\title{
Detection and Tracking Volumes of Interest in 3D Printed Tissue Engineering Scaffolds using 4D Imaging Modalities
}

\author{
A. I. Kondarage ${ }^{1}$, B. Gayani ${ }^{1}$, G. Poologasundarampillai ${ }^{2}$, A. Nommeots-Nomm ${ }^{3}$, P.D. Lee ${ }^{4}$, \\ T.D. Lalitharatne ${ }^{5}$, N.D. Nanayakkara ${ }^{6}$, J.R. Jones ${ }^{3}$ and A. Karunaratne ${ }^{1}$
}

\begin{abstract}
Additive manufacturing (AM) platforms allow the production of patient tissue engineering scaffolds with desirable architectures. Although AM platforms offer exceptional control on architecture, post-processing methods such as sintering and freeze-drying often deform the printed scaffold structure. In situ 4D imaging can be used to analyze changes that occur during post-processing. Visualization and analysis of changes in selected volumes of interests (VOIs) over time are essential to understand underlining mechanisms of scaffold deformations. Yet, automated detection and tracking of VOIs in 3D printed scaffolds over time using 4D image data is an unsolved image processing task. This paper proposes a new image processing technique to segment, detect and track volumes of interest of 3D printed tissue engineering scaffolds. The method is validated using a 4D synchrotron sourced micro-CT image data captured during the sintering of bioactive glass scaffolds in situ. The proposed method will contribute to developing patientspecific scaffolds with better controllable designs and optimum properties.
\end{abstract}

\section{INTRODUCTION}

Additive manufacturing (AM) is currently in a great interest in tissue engineering due to its ability to fabricate geometrically sophisticated structures. This tailor-made manufacturing process can be used to produce patient-specific tissue scaffold upon further development [1] The mechanical and biological properties of scaffolds should be considered beforehand to develop such scaffolds. Hence, pore size, strut thickness and the interconnection of pores which are crucial for cell proliferation as well as mechanical strength should be predetermined [2]. Designing scaffold architectures are one way to achieve the desired biological and mechanical properties. Depending on the biomaterial choice, architectures such as woodpile, and honeycomb are used to infill the

*This research was funded by the National Institute for Health Global Health Research (grant number 1613745: NIHR Group on POsT Conflict Trauma ; PrOTeCT). GP acknowledges funding from the EPSRC grant EP/M023877/1 and PDL acknowledges funding from the MRC ImagingBioPro grant (MR/R025673/1), the Research Complex at Harwell and Royal Academy of Engineering (CiET1819/10). The Imaging was performed on I13-2 of the Diamond Light Source synchrotron (MT13241).

${ }^{1}$ A.I. Kondarage, B. Gayani and A. Karunaratne are with the Department of Mechanical Engineering, University of Moratuwa, Moratuwa, Sri Lanka.(e-mail: k.achintha.i@ieee.org)

${ }^{2}$ G. Poologasundarampillai is with School of Dentistry, Institute of Clinical Sciences, University of Birmingham, Birmingham, United Kingdom

${ }^{3}$ A. Nommeots-Nomm and J.R. Jones are with the Department of Materials, Imperial College London, London, United Kingdom

${ }^{4}$ P.D. Lee is with the Department of Mechanical Engineering, University College London, London, United Kingdom.

${ }^{5}$ T.D. Lalitharatne is with Dyson School of Design Engineering, Imperial College London, London, United Kingdom.

${ }^{6}$ N.D. Nanayakkara is with the Department of Electronic and Telecommunication, University of Moratuwa, Moratuwa, Sri Lanka.

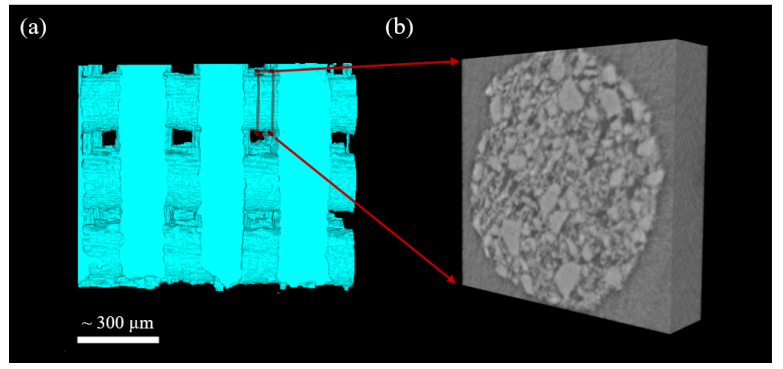

Fig. 1. (a) shows a segmented 3D image of the scaffold (b) shows a Volume of Interest required to track.

scaffold [3]. Here, the geometries are aligned with linear pore connections to facilitate cell proliferation while maintaining continuous material in the $\mathrm{z}$-direction to enhance the mechanical strength [4]. Woodpile architecture is the simplest yet an exemplification of a true scaffold structure those have homogenously arranged pores providing a convenient path to allow the blood pass, high load-supporting ability and excellent mechanical properties [5].

One of the major problems when designing 3D printed patient specific scaffolds is alterations occur during the manufacturing process those ultimately result in desperate final output to the designed CAD model [6]. Additive manufacturing of ceramics and glass scaffolds usually involves the sintering of green body scaffolds. Sintering delivers a densified scaffold with better mechanical properties, and because of this densification, a global level 3D shrinkage of the scaffold can be observed [4]. Hydrogels also show a significant swelling when the crosslinking density is lowered to obtain improved endothelial network formation [7]. In post processing events such as freeze-drying, the scaffolds show different ratios of swelling and pore sizes [8].

In situ 4D imaging methods such as synchrotron-sourced micro-computed tomography (micro- CT) can be used to characterize deformations occurs during the manufacturing of tissue engineering scaffolds. Struts can be categorized as building elements of a scaffold. Therefore, four-dimensional analyzing dynamics of struts and unit cells of scaffolds is important to determine the optimal processing conditions and understand the underlying mechanisms. For example, recent study of Nommeots-Nomm et al discusses influence of particle densification within a strut for the global level structural changes of 3D printed bioactive glass scaffolds[4].

Image analysis plays a vital role in the quantitative analy- 


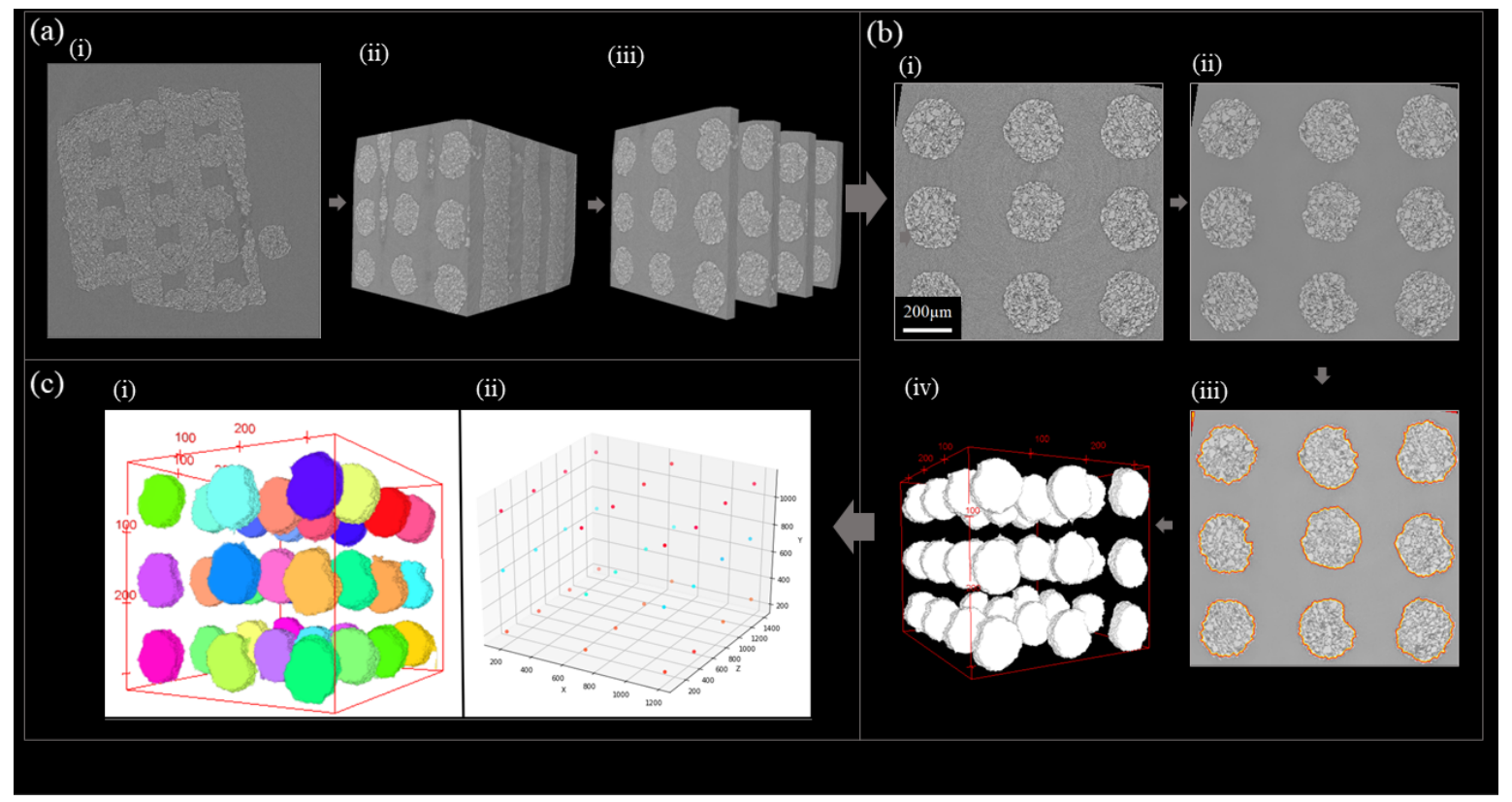

Fig. 2. Image processing pipeline to detect VOIs; (a) Pre-processing (i) Image slice of a reconstructed 3D image (ii) cropped, rotated and resampled 3D image (iii) 3D image generated with image slices which include VOIs; (b) Noise reduction and segmentation of VOIs (i) Image slice before the noise reduction (ii) Image slice after the noise reduction (ii) Implicit Contour fitted to Regions of Interest (iii) Segmented 3D image;(c) VOI detection

sis of 3D printed scaffolds. Implementation of customized image processing methods is important to analyze postprocessing events of 3D printed scaffolds over time. One of the challenging image processing tasks is to track volumes of interests (VOIs) of scaffolds such as slices of struts and unit cells over time in a series of 3D images. In this work, we propose an image processing method to detect and track VOIs in 3D printed tissue engineering scaffolds using 4D image data for the first time. A 4D synchrotronsourced micro-tomography image dataset captured during the sintering of bioactive glass scaffolds was used to demonstrate the image processing method.

\section{Methods}

The proposed image processing method is implemented incorporating ImageJ API, and Skimage, Sklearn python libraries and can be accessed via github (link). The method is composed of several important steps, including preprocessing, segmentation, VOI detection and VOI tracking.

\section{A. Image acquisition}

The scaffolds of bioactive glass ICIE16 (49.46 mol.\% $\mathrm{SiO}_{2}, 36.27 \mathrm{~mol} . \% \mathrm{CaO}, 6.6 \mathrm{~mol} . \% \mathrm{Na}_{2} \mathrm{O}, 1.07 \mathrm{~mol} . \%$ $\mathrm{P}_{2} \mathrm{O}_{5}$ and $6.6 \mathrm{~mol} . \% \mathrm{~K}_{2} \mathrm{O}$ ) were produced according to the protocol presented by Nommeots-Nomm et al [9]. Here, green body scaffolds with wood pile architecture were produced. The size of the scaffolds was $1.8 \mathrm{~mm} \times 1.8 \mathrm{~mm} \times$ $1.8 \mathrm{~mm}$. The 3D printed green body scaffolds were sintered to obtain fully densified glass scaffold. The sintering was performed in the bespoke proportional- integral- derivativecontrolled 'Laura' furnace[4] and imaged in-situ using synchrotron sourced X-ray microtomography at the DiamondManchester Imaging Branchline I13-2 of Diamond Light
Source. The imaging was conducted with a filtered pink polychromatic beam in the energy range of 8 to $30 \mathrm{KeV}$ and the emitted X-ray beam was captured using a CMOS detector with a resolution of $2560 \times 2160$. All scans were captured with a total magnification of $4 \times$ which resulted in an effective isotropic pixel size of $0.81 \mu \mathrm{m}$. Projections captured during the sintering of the scaffold were reconstructed using Filtered Back Projection algorithm. Here, 93 3D reconstructed images captured over time ( 3 hours) were considered to validate the proposed image processing technique.

\section{B. Volume of interest}

Fig.1.a shows a segmented image of green body of the scaffold obtained from a 3D reconstructed scan. To understand the changes occur within a strut of the scaffold, slices of struts (Example: Fig.1.b) which are considered as the volumes of interest (VOIs) can be taken into the analysis. Prior to track the VOIs in 4D, VOIs should be automatically detected.

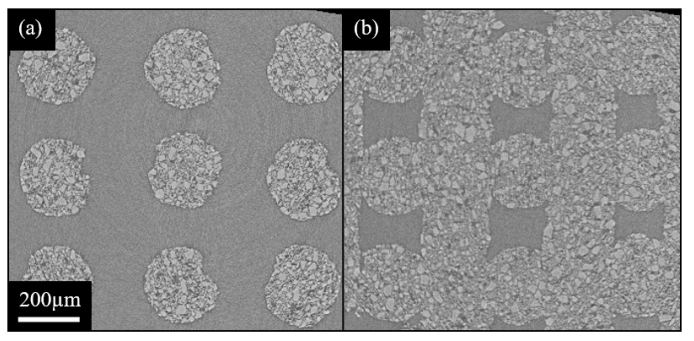

Fig. 3. (a) shows an image slices which include sections VOIs. (b) shows image slices which do not include sections of VOIs. 


\section{Preprocessing of Images}

Size of a $3 \mathrm{D}$ reconstructed image is $2560 \times 2560 \times 2159$. Fig. 2. a. i shows a slice of a $3 \mathrm{D}$ reconstructed image. As the initial step of pre-processing each 3D image was rotated as such struts are aligned in $\mathrm{z}$ and $\mathrm{y}$ direction of the image coordinates. Then the images were cropped to the image size of $1356 \times 1296 \times 1540$ to remove excess background of images. All the images were resampled with a factor of 0.25 to reduce computational cost required to detect VOIs. Fig. 2. a. ii shows a resampled 3D image.

As a pre-processing step, image slices without VOIs were removed using a machine learning classifier. Here, each image slice of a 3D image was classified into 2 classes. One class is slices which include pixels of VOIs (Example: Fig. 3. a) and the other class is slices which do not include pixels VOIs (Example: Fig. 3. b). A set of labelled images of these 2 classes were used as training data to train a Support Vector Machine (SVM) Classifier.

The SVM classifier was implemented using Scikit-learn [10]. The set of pixel values of a further resampled image slice $(64 \times 64)$ was considered as the feature vector of that particular image slice. The set of hyperparameters of the SVM model is $\{\mathrm{C}: 1.0$, shape of the function : onevs-rest(ovr), kernel : RBF, gamma: scale (1 / (number of features $\times$ variance of input features ) $\}$. The trained classifier was used to detect image slices which include VOIs. Then image slices which don't include VOIs were removed. An example is shown in Fig.2. a. iii.

\section{Noise reduction and segmentation of VOIs}

As a pre-processing step for the segmentation of strut cross sections, nonlocal mean filtering was applied to reduce the background noise of the image (Fig. 2. b. ii). Non-local means algorithm uses redundant information of the image to reduce the noise by performing a weighted average of pixel values considering spatial and intensity similarities between pixels. It is calculated between equally sized patches as they capture geometry and texture around the site in consideration [11].

Level set algorithm [12] was used to segment strut crosssections in each image slice with VOIs. Here a contour embedded as a zero level of a higher dimension function named as level set function is fitted to boundaries of regions of interest (ROIs). Zero level set (contour) can be described using the Equation (1) for a given level set function $\phi(x, y, t)$ where $\phi(x, y, t)$ is surface in 3 dimensions. In this case, the level set function evolves until it reaches the boundaries of ROIs. The movement formula for the level set can be formulated as Equation (2). $F$ is the speed function. In this case, $F$ is related to the edges of the image.

$$
\begin{gathered}
\phi(x, y, t)=0 \\
\phi_{t}+F|\Delta \phi|=0
\end{gathered}
$$

Fig. 2. b. iii shows the fitted contour to boundaries of struts of the scaffolds. Fig. 2. b. iv shows the segmentation of VOIs in the $3 \mathrm{D}$ image.

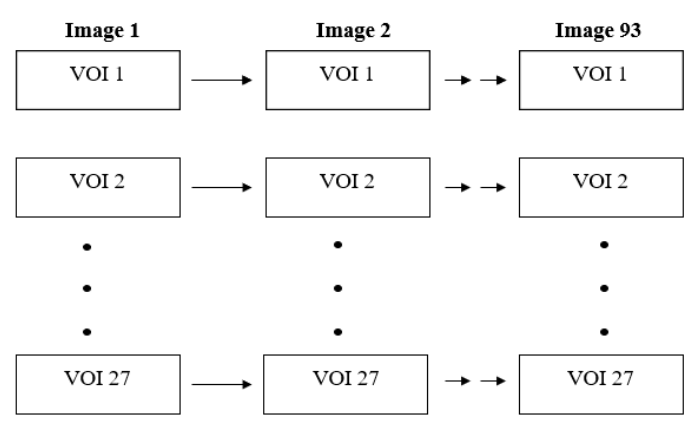

Fig. 4. Tracking VOIs over the image series

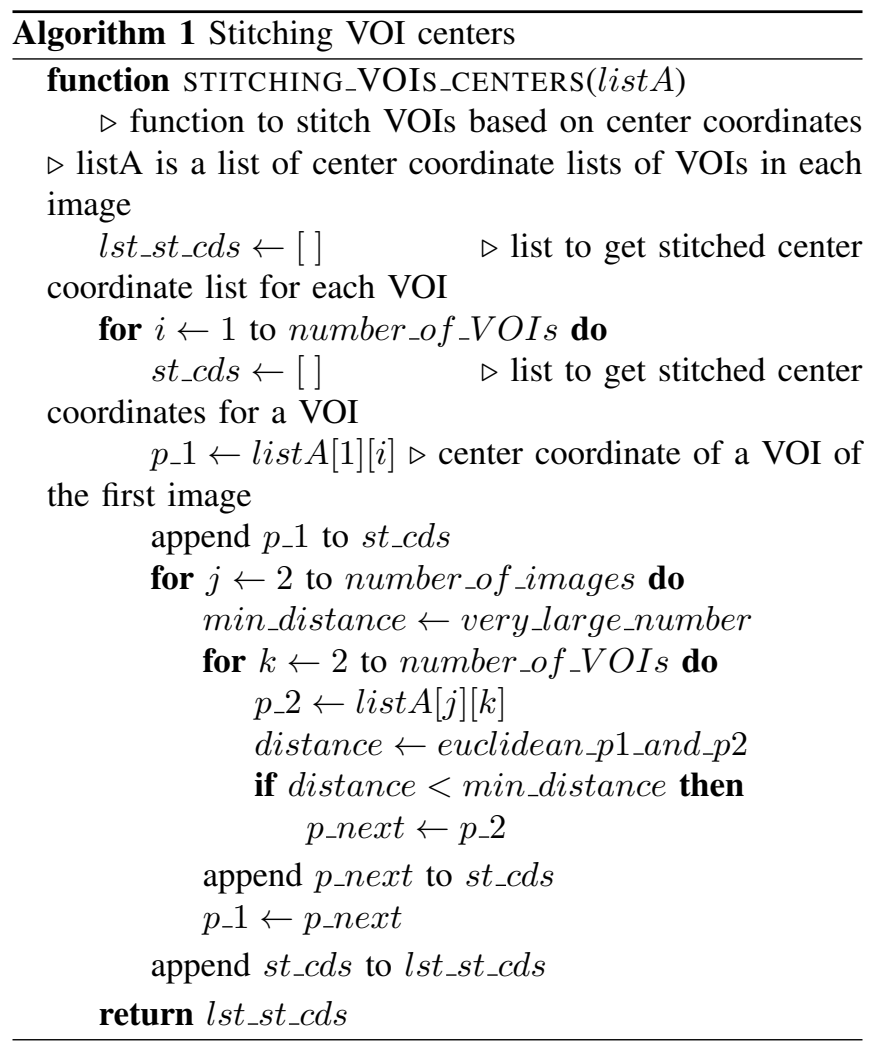

\section{E. Detecting VOIs}

After the segmentation, each VOI can be identified as separated connected components of the 3D image. Each of these connected components were labelled using scikit-image [13] Python library. A 3D image with labelled VOI is shown in the Fig. 2. c. i Centre of the VOI were obtained using regionprops function of scikit-image. Fig. 2. c. ii shows center coordinates obtained by this method. Based on these center coordinates of VOIs in the original image (before resampling) can also be obtained. In this work VOIs (strut slices) with the dimension of $150 \times 140 \times 70$ were generated at each time-time point (for each 3D image).

\section{F. Tracking VOIs}

VOI detection method delivers center points respective to VOIs in all the captured 3D images. In this work more than 


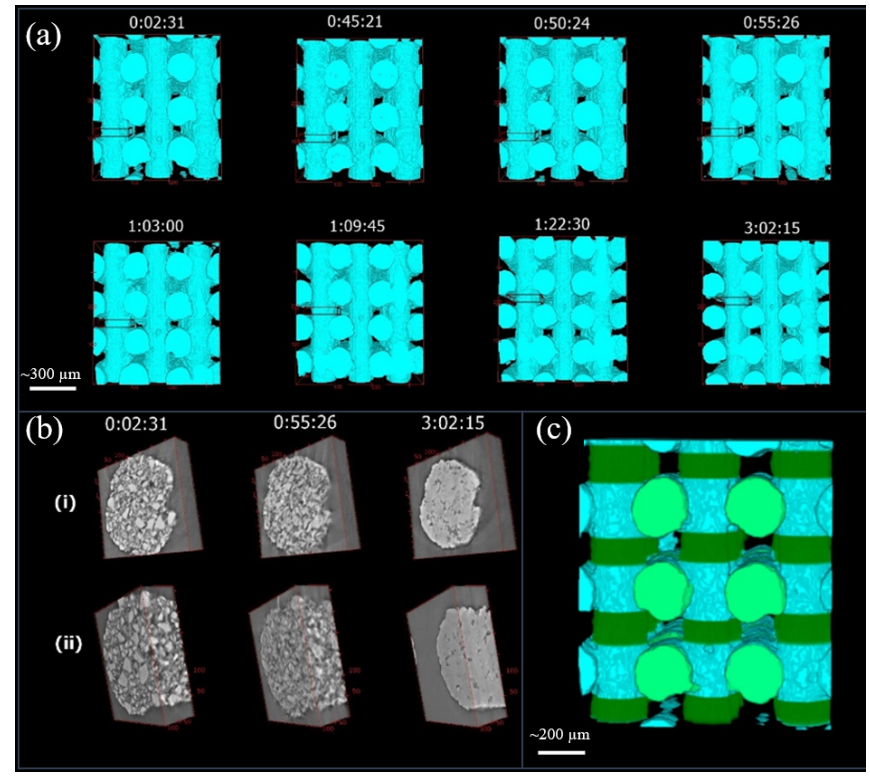

Fig. 5. (a) Tracking VOIs of scaffold over time; (b)Child volumes/ VOIs obtained over time (i) based on coordinates obtained from proposed method.and (ii) based on fixed coordinates; (c) Segmented image of VOIs within a 3D printed scaffold designed with wood-pile architecture.

27 VOIs were detected in each 3D image (Fig. 2 C. i). This work aims to track a particular VOI over the series of scans (Fig. 5). Here, the center coordinates VOIs in consecutive scans were matched based on minimum Euclidean distance, assuming that movement of a VOI within 2 consecutive scan is less than the distance within different VOIs. The pseudo code for VOI stitching over-time is shown in algorithm 1.

\section{RESUlTS AND Discussion}

The average center error of the proposed tracking algorithm is 2.8 , which is corresponding to the distance of 3 (rounded) voxels and $2.43 \mu \mathrm{m}$. Fig. 5. a illustrate the tracking of VOI overtime with the deformations occurs in the overall structure of the scaffold.

One of the main advantages of tracking a VOI is visualization of changes occurred within the VOI over-time. Fig. 5.b illustrates the visualization of a strut over time with tracking algorithm (Fig.5.b.i) and fixed image coordinates (Fig.5.b.ii ). Child volumes obtained from the tracking algorithm can also be used as a pre-processing step for further quantitative analysis. A video generated by tracking a VOI over the complete scan set can be access via github (ink).

The proposed algorithm is not limited to detect and track VOIs (strut slices) over the z-direction of the scaffold. It can also be applied be applied to struts in y direction simply by rotating the images. Fig.5.c shows the segmented VOIs within a 3D printed scaffold design with woodpile architecture that can be detected and tracked based on the proposed method.

\section{Conclusions And Future Directions}

This paper proposed a novel image processing technique to segment, detect, and track volumes of interest in 3D printed tissue engineering scaffolds using 4D imaging modalities. The proposed method was validated using 4D synchrotron sourced micro-CT image data captured during the sintering of bioactive glass scaffolds in situ. We believe that the proposed VOI tracking algorithm will contribute to the dynamic characterization of $3 \mathrm{D}$ printed scaffolds and realization of patient specific scaffolds. As medical implants are required to confirm quality assurance, understanding deformations occur in the 3D printed scaffold is vital. Therefore, dedicated image analysis frameworks to characterize tissue engineering scaffolds are necessary. The proposed image processing methodology can be integrated into such image analysis frameworks.

\section{REFERENCES}

[1] S. Bose, S. Vahabzadeh, and A. Bandyopadhyay, "Bone tissue engineering using 3D printing," Mater. Today, vol. 16, no. 12, pp. 496-504, 2013.

[2] S. Li, F. Tallia, A. A. Mohammed, M. M. Stevens, and J. R. Jones, "Scaffold channel size influences stem cell differentiation pathway in 3-D printed silica hybrid scaffolds for cartilage regeneration," Biomater. Sci., vol. 8, no. 16, pp. 4458-4466, 2020.

[3] L. C. Hsiao, A. Z. M. Badruddoza, L. C. Cheng, and P. S. Doyle, "3D printing of self-assembling thermoresponsive nanoemulsions into hierarchical mesostructured hydrogels," Soft Matter, vol. 13, no. 5, pp. 921-929, 2017.

[4] A. Nommeots-Nomm et al., "Four-dimensional imaging and quantification of viscous flow sintering within a 3D printed bioactive glass scaffold using synchrotron X-ray tomography," Mater. Today Adv., vol. 2, p. 100011, 2019.

[5] E. Cuan-Urquizo, F. Shalchy, and A. Bhaskar, "Compressive stiffness of staggered woodpile lattices: Mechanics, measurement, and scaling laws," Int. J. Mech. Sci., vol. 187, no. July, p. 105932, 2020.

[6] M. Qasim, D. S. Chae, and N. Lee, "Advancements and frontiers in nano-based $3 \mathrm{~d}$ and $4 \mathrm{~d}$ scaffolds for bone and cartilage tissue engineering," Int. J. Nanomedicine, vol. 14, pp. 4333-4351, 2019.

[7] A. Brown, H. He, E. Trumper, J. Valdez, P. Hammond, and L. G. Griffith, "Engineering PEG-based hydrogels to foster efficient endothelial network formation in free-swelling and confined microenvironments," Biomaterials, vol. 243, no. February, 2020.

[8] X. Luo et al., "Thermo/photo dual-crosslinking chitosan-gelatin methacrylate hydrogel with controlled shrinking property for contraction fabrication," Carbohydr. Polym., vol. 236, no. December 2019, p. 116067, 2020.

[9] A. Nommeots-Nomm, P. D. Lee, and J. R. Jones, "Direct ink writing of highly bioactive glasses," J. Eur. Ceram. Soc., vol. 38, no. 3, pp. 837-844, 2018.

[10] F. Pedregosa et al., "Scikit-learn: Machine Learning in Python," J. Mach. Learn. Res., vol. 12, no. 85, pp. 2825-2830, 2011.

[11] A. Buades, B. Coll, and J. Morel, "Non-Local Means Denoising," Image Process. Line, vol. 1, pp. 208-212, 2011.

[12] R. Malladi, J. A. Sethian, and B. C. Vemuri, "Shape Modeling with Front Propagation: A Level Set Approach," IEEE Trans. Pattern Anal. Mach. Intell., vol. 17, no. 2, pp. 158-175, 1995.

[13] S. van der Walt et al., "scikit-image: image processing in Python," PeerJ, vol. 2, p. e453, 2014.

[14] D. Wang, H. Lu, and M. H. Yang, "Online object tracking with sparse prototypes,” IEEE Trans. Image Process., vol. 22, no. 1, pp. 314-325, 2013.

[15] F. Yang, H. Lu, and M. H. Yang, "Robust superpixel tracking," IEEE Trans. Image Process., vol. 23, no. 4, pp. 1639-1651, 2014. 